\title{
Reply to Letter "NOCTP and NRCT Are Not Complete without Tailored Motivational Communication"
}

\author{
Vita Machiulskiene ${ }^{a}$ Bente Nyvad $^{b}$ \\ ${ }^{a}$ Clinic of Dental and Oral Pathology, Faculty of Odontology, Lithuanian University of Health Sciences, \\ Kaunas, Lithuania; ${ }^{\mathrm{b}}$ Section for Oral Ecology and Caries Control, Department of Dentistry and Oral Health, \\ Aarhus University, Aarhus, Denmark
}

\section{Dear Editor,}

We thank the authors for their comments on our article [Machiulskiene et al., 2020], and for opening a relevant discussion about differences in opinions regarding dental caries and dental caries-related definitions. They came up with several critical points: a missing comparison of our definitions with previously published terms and concepts [Mertz-Fairhurst et al., 1998; Mijan et al., 2014; Innes et al., 2016], and missing the patient perspective as well as lacking priority for professionally guided self-care in the descriptions of some of our terms.

While we embrace this criticism and support continued discussion among peers, we would like to point out that it was not the aim of our report to discuss potential differences in caries terminology between our consensus statements and any other previous attempts to define the terms associated with dental caries and related matters. Consensus reports are not classic scientific communications with lengthy discussions; they represent expert opinions only. Indeed, the absence of discussion could be considered a major limitation of such reports. Consensus statements are typically developed in conjunction with a consensus conference, at which a pre-selected topic is debated by a panel of experts, followed by anonymous voting. The collective opinion of the panel is expressed as a percentage agreement, which, in our report, was based on

karger@karger.com

(c) 2021 S. Karger AG, Basel

www.karger.com/cre

Karger! the Delphi method with an a priori established threshold of $80 \%$ defining "consensus" [Beighton, 2017]. Consensus statements do not give "specific algorithms or guidelines for practice," rather, they aim to represent "state of the art" knowledge, and should thus be re-evaluated from time to time [Beighton, 2017]. Nevertheless, the most relevant publications about caries terms, including the paper by Innes et al. [2016], are cited in our paper.

The authors of the letter are particularly concerned about our descriptions of non-operative caries treatment/ management/control/care (NOCTP) and non-operative caries/cavity treatment (NRCT), which they find are incomplete as they do not highlight the use of tailored motivational communication. In our consensus report, NOCTP was a broad definition that included all aspects of the non-operative treatment of caries. We agree with the authors of the letter that the education of patients is an essential part of non-operative caries treatment, as was demonstrated nearly 30 years ago by the pioneering work of Carvalho et al. [1992]. However, a structured protocol including proper lesion detection, diagnosis of lesion activity, the identification and modulation of caries-accelerating factors, and longitudinal follow-up may be equally important for the outcome.

In our report, the multifactorial treatment approach is further supported by a reference showing that when non- 
operative cavity treatment has a strong focus on the dialogue with the patient, this may reduce the need for operative intervention and empower parents and children to participate actively in decision-making and self-care [Hansen and Nyvad, 2017]. In retrospect, we might have placed more weight on personalized communication as an integrated part of the delivery of professional caries control. The multifactorial treatment approach implies that the focus is always on the patient and the goal is to help the patient to maintain his/her dentition in good health.

On behalf of the authors, Vita Machiulskiene Bente Nyvad

\section{Conflict of Interest Statement}

The authors have no conflicts of interest to declare.

\section{Author Contributions}

V.M. and B.N. drafted the text. All authors of the consensus statements critically reviewed and approved the final version of the text.

\section{References}

Beighton D. Consensus Statements. Caries Res. 2017;51(5):I-II.

Carvalho JC, Thylstrup A, Ekstrand KR. Results after 3 years of non-operative occlusal caries treatment of erupting permanent first molars. Community Dent Oral Epidemiol. 1992 Aug; 20(4):187-92.

Hansen NV, Nyvad B. Non-operative control of cavitated approximal caries lesions in primary molars: a prospective evaluation of cases. J Oral Rehabil. 2017 Jul;44(7):537-44.
Innes NP, Frencken JE, Bjørndal L, Maltz M, Manton DJ, Ricketts D, et al. Managing carious lesions: consensus recommendations on terminology. Adv Dent Res. 2016 May;28(2): 49-57.

Machiulskiene V, Campus G, Carvalho JC, Dige I, Ekstrand KR, Jablonski-Momeni A, et al. Terminology of Dental Caries and Dental Caries Management: Consensus Report of a Workshop Organized by ORCA and Cariology Research Group of IADR. Caries Res. 2020;54(1):7-14.
Mertz-Fairhurst EJ, Curtis JW Jr, Ergle JW, Rueggeberg FA, Adair SM. Ultraconservative and cariostatic sealed restorations: results at year 10. J Am Dent Assoc. 1998 Jan;129(1): $55-66$.

Mijan M, de Amorim RG, Leal SC, Mulder J, Oliveira L, Creugers NH, et al. The 3.5-year survival rates of primary molars treated according to three treatment protocols: a controlled clinical trial. Clin Oral Investig. 2014 May;18(4):1061-9. 